\title{
Understanding Radiation Damage to Cells Using Microbeams
}

\author{
M. FolkArd ${ }^{a}$, K.M. Prise ${ }^{a}$, C. $\mathrm{ShaO}^{a}$, S. Gilchrist ${ }^{a}$, \\ G. Schettino ${ }^{a}$, A.G. Michette ${ }^{b}$ And B. Vojnovic ${ }^{a}$ \\ ${ }^{a}$ Gray Cancer Institute, Mount Vernon Hospital, P.O. Box 100 \\ Northwood, HA6 2JR, UK \\ ${ }^{b}$ King's College London, Strand, London WC2R 2LS, UK \\ Cellular micro-irradiation techniques provide unique experimental op- \\ portunities for understanding how ionizing radiation interacts with living \\ cells and tissues. Using microbeams, it is possible to deliver precise doses of \\ radiation to selected individual cells, or sub-cellular targets in vitro. This \\ technique continues to be applied to the investigation of a number of phe- \\ nomena currently of great interest to the radiobiological community. In \\ particular, it is the study of so-called "non-targeted" effects (where cells are \\ seen to respond indirectly to ionizing radiation) that are benefiting most \\ from the use of microbeam approaches.
}

PACS numbers: 41.75.Ak, 87.50.--a

\section{Introduction}

The Gray Cancer Institute (GCI) has been involved in the development and radiobiological application of both ion microbeams [1] and focused X-ray microprobes [2] for a number of years. Microbeams continue to be a powerful research tool in studies of radiobiolical effects at low doses, as they can be used to deliver exact low doses of radiation to selected individual cells and the subsequent damage can be analysed on a cell-by-cell basis. There is increasing evidence that so-called "non-targeted" effects have a significant influence at low doses. Non-targeted effects are those where cells are seen to respond indirectly to ionizing radiation and are in conflict with the conventional view of cellular radiation damage, which assumes that direct damage to the DNA helix is necessary to induce critical effects [3]. One important non-targeted effect is the "bystander effect" where it is observed that unirradiated cells exhibit damage in response to signals transmitted by irradiated neighbours [4]. The ability of the microbeam to selectively target individual cells within a cell population has made this a highly sought-after technique 
for studying the bystander effect. Consequently, the number of groups actively involved in the development of radiobiological microbeams has risen dramatically in the past few years, from just four or five in the 1990s, to around thirty in the past year. Despite this, the number of facilities in routine use remains low, reflecting in part the difficulty in solving a range of technical issues that arise when applying this technique to living cells. The GCI microbeams have been fully operational for a number of years and are used extensively for investigating non-targeted effects in both cell and tissue models. Through our studies, we have been able to gain some insight into both the magnitude of these effects and the mechanisms that underpin them.

\section{Anomalous low-dose effect in cells}

The phenomenon now called the bystander effect was first reported by Nagasawa and Little [5], who observed chromosome damage in $30 \%$ of cells following exposure to a broad field of $\alpha$-particles such that only $1 \%$ of cell nuclei are actually hit. While it is the bystander effect that has attracted most interest, a number of other non-targeted and anomalous low-dose effects have been reported $[3,6]$. These include adaptive responses, low-dose hypersensitivity, genomic instability, the inverse dose-rate effect and the up, or down regulation genes at doses below levels of significant of DNA damage. One reason for the interest in these phenomena is that they potentially undermine current estimates of the risk associated with exposure to occupational and environmental levels of radiation. At these levels, reliable data from epidemiology or experiments are not currently available, so an estimate is made, based on a linear extrapolation of known risks at higher doses to the low-dose region (the so-called "linear no-threshold" model). Non-targeted effects are also of potential relevance to the advancement of the treatment of cancer by radiotherapy. One possibility being considered is to increase the "therapeutic benefit" by selectively modifying the response of either the tumour, or the healthy tissue to radiation by chemical action directed at the signalling molecules involved in the bystander effect.

\section{Methods for micro-irradiating cells}

A number of ionizing radiations are suitable for use as a microbeam. To date, radiobiolical microbeams have been developed using charged particles [7], low energy X-rays (up to $\approx 10 \mathrm{kV}$ ) [2] and low energy electrons (up to $\approx 25 \mathrm{kV}$ ) [8]. There are important reasons for using radiations with different qualities; with regard to environmental exposures, an individual cell will typically see roughly one electron traversal per year and about one $\alpha$-particle traversal per century. However, the dose to the cell by a single electron is about $1 \mathrm{mGy}$, and that following an $\alpha$-particle traversal is typically several hundred times greater, so the overall effect of both electrons and particles is significant. Environmental exposure to $\alpha$-particles arises mainly through inhalation of radon, leading to the exposure 
of lung epithelial cells. From a microdosimetric viewpoint, it can be shown that most lung cells are not irradiated, but those that are hit will be unlikely to receive more than one $\alpha$-particle traversal. Thus there is the potential for a bystander effect resulting from a single $\alpha$-particle traversal of single cell. Consequently, most particle microbeams use light ions (either protons or helium ions) as they are of greatest radiobiolical relevance, however, studies using heavier ions are also of interest in connection with particle radiotherapy using ions other than the protons and in studies of the risks associated with long-term space travel. Heavy ions also have the advantage that they are less easily scattered.

To study the much lower doses that occur when an electron traverses a cell requires the use of an X-ray or electron microbeam. Also, low-energy X-rays are the source of choice for achieving the finest probes. This is because unlike particles and electrons, they interact almost entirely through the photoelectric effect and therefore are not scattered. The "fineness" on an X-ray probe is ultimately limited by the range of the secondary electrons it sets in motion. For $1.48 \mathrm{keV} \mathrm{Al}_{K} \mathrm{X}$-rays this is about $50-70 \mathrm{~nm}$, while for $0.28 \mathrm{keV} \mathrm{C} \mathrm{C}_{K}$ X-rays it is less than $10 \mathrm{~nm}$. By contrast, a low-energy light ion will be significantly scattered by the vacuum exit window, the transmission detector (if used) and the cell itself, such that probe sizes less than 1-2 $\mu \mathrm{m}$ are difficult to achieve, irrespective of the focussing or collimation method used. Electron microbeams, while relatively straightforward to develop, are the least favourable with regard to probe size. This is because the electron energies required to penetrate the cell $(>15 \mathrm{keV})$ produce secondary electrons within the cell with ranges of several microns.

Historically, the first successful microbeams to operate routinely used collimated, charged particles. More recently, several facilities have been developed that are an adaptation of an existing micro-irradiation facility, such as an analytical microprobe. Examples of those that use collimation are our own facility at the Gray Cancer Institute, the Radiological Research Accelerator Facility (RARAF), Columbia University, New York and the heavy ion facility at the Japan Atomic Energy Research Institute, Takasaki, Japan. Examples of focussed facilities are those being developed at GSI, Darmstadt and CENBG, Bordeaux and a new facility at RARAF. See [7] for a comparison of the different approaches used to develop particle microbeams.

\section{The GCI charged-particle microbeam}

The GCI charged-particle microbeam has been in use since the mid-1990s and its development has been reported previously $[1,9]$. A fine radiation beam is formed using a $1 \mu \mathrm{m}$ diameter bore fused silica capillary collimator, mounted at the end of a vertical particle beamline. The source of radiation is a $4 \mathrm{MV}$ VdG accelerator, producing either protons, or ${ }^{3} \mathrm{He}^{2+}$ ions, that are transported upward through the floor of the microbeam laboratory to the cell irradiation apparatus, mounted on an optical table. Let us note that ${ }^{3} \mathrm{He}^{2+}$ ions are radiobiologically 

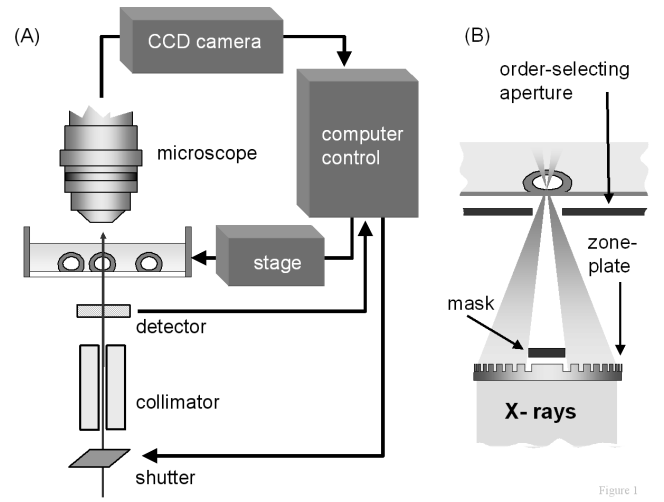

Fig. 1. Schematic diagram of (A) the charged-particle microbeam and (B) the method used in the X-ray microprobe to focus X-rays (not to scale). The facilities use similar automated arrangement for finding and aligning cells, as indicated in (A).

equivalent to ${ }^{4} \mathrm{He}^{2+}$ ions of the same ionization density, but have greater penetration. A schematic diagram of the charged-particle microbeam is depicted in Fig. 1A.

The cells are attached to a thin plastic membrane that forms the base of a cell dish containing cell culture medium. The dish is located on a 3-axis micropositioning stage above the collimator. During irradiation, each cell is located, in turn, above the collimator and exposed to an exact, predefined number of particles. These are counted and controlled using a photomultiplier tube mounted just above the cell dish. When a particle traverses, the photomultiplier tube detects the pulse of light from a thin scintillator "sandwiched" between the collimator exit and the underside of the cell dish.

Measurements show [10] that using ${ }^{3} \mathrm{He}^{2+}$ ions $99 \%$ of cells are targeted with an accuracy of $\pm 2 \mu \mathrm{m}$. When single particle counting, the detection efficiency is greater than $99 \%$, with no missed particles and less than $1 \%$ false positives. For some studies, it is necessary to individually irradiate many thousands of cells per dish. It is essential therefore that the process of target identification, alignment and irradiation are both automated and rapid. Therefore the GCI facility has advanced cell recognition and alignment capabilities such that up to 10,000 cells per hour can be individually located and irradiated. The cell alignment system makes us of an epi-fluorescent microscope to view stained cells supported on a computer-controlled, 3-axis micropositioning stage.

\section{The GCI soft X-ray microprobe}

The production of fine X-ray probes can now be achieved by the use of X-ray optics developed for high-resolution X-ray microscopic imaging. The finest X-ray probes have been obtained using "zone plates". These are circular diffraction gratings with radially increasing line density, such that diffracted X-rays are brought 
to an axial focus. The zone plate is mounted in an assembly with an arrangement of masks and apertures to ensure that only first-order diffracted X-rays reach the target (see Fig. 1B).

$\mathrm{X}$-rays are generated using a tabletop microfocus source [2]. Characteristic X-rays of carbon $(278 \mathrm{eV})$, aluminium $(1.49 \mathrm{keV})$, or titanium $(4.5 \mathrm{MeV})$ are generated by focused electron bombardment of a thick target. The electrons are generated by an electron gun operated at $10-15 \mathrm{kV}$ with respect to the target. In addition to characteristic radiation, the electron bombardment of the target will produce a continuum of bremsstrahlung radiation, which is undesirable because it will not be correctly focused by the zone plate. This is reduced by reflecting the radiation off a $25 \mathrm{~mm}$ diameter silica mirror mounted between the carbon target and the zone-plate focusing assembly. To locate and align cells, an identical system to that installed on the particle microbeam is used. In use, using carbon X-rays, it is possible to achieve dose-rates around $1-2 \mathrm{~Gy} \mathrm{~s}^{-1}$ and spot sizes of about $1 \mu \mathrm{m}$.

\section{Studies of the bystander effect using the GCI microbeam}

In an initial study of the bystander effect, a population of $\approx 800$ cells (primary human fibroblasts) was seeded onto a cell dish and the nucleus of one cell was targeted with between 1 to 15 counted ${ }^{3} \mathrm{He}^{2+}$ ions [11]. The amount of damage was assessed by scoring the formation of micronuclei throughout the cell population. Micronuclei are small extra nuclei expressed in damaged cells during the first cell division. On average, a 2-3-fold increase over background was observed when just a single cell is targeted. It was also seen that the level of damage was independent of the number of particles traversing the cell, suggesting that a single helium ion is sufficient to induce a full bystander response. Another observation is that the bystander response is always seen when this experiment is performed, from which it can be concluded that every cell within the population can produce a bystander signal, but not every cell will respond to the signal.

The bystander response has also been observed using our soft X-ray microprobe and because it is possible to deliver much lower doses using this facility (using particles, the dose to a cell cannot be less than that deposited by one ion), the very low-dose region has been studied in detail. Schettino et al. [12] have used the X-ray microprobe to carefully explore the bystander effect at doses between 0.05 Gy and 0.2 Gy and have observed a tendency for cell populations to exhibit either the full bystander effect (i.e. about $5-7 \%$ cell kill) or no effect above background, with a greater probability of full effect at higher doses (see Fig. 2). This suggests that rather than exhibiting a gradual dose-effect between zero dose and saturation (which occurs at about $0.2 \mathrm{~Gy}$ ), the bystander effect is triggered to maximum effect once a threshold dose (which may vary slightly from cell to cell) is exceeded. Schettino et al. [13] have also analysed the probability of a cell exhibiting a media-borne bystander response as a function of the distance from the irradiated cell in a non-confluent cell population and find no correlation with 


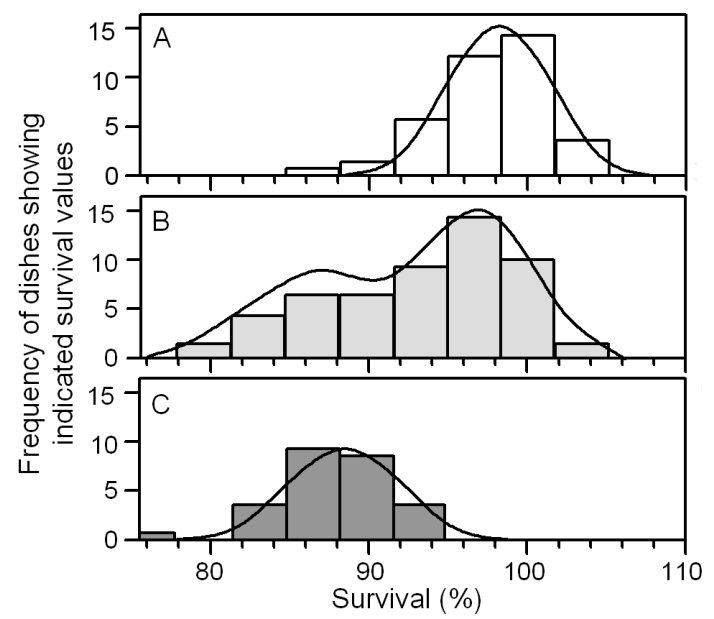

Fig. 2. Histogram of the distribution of bystander survival values after irradiating a single cell with focused X-rays for control (A), low-dose, below 0.3 Gy (B) and high-dose (C) data. The lines are for guidance only. The low-dose data is reasonably described by the sum of two Gaussian distributions, indicating a binary "all-or-nothing" bystander response. Data redrawn from [12].

distance up to $3 \mathrm{~mm}$ and with an average distance between cells of $150 \mu \mathrm{m}$. However, the distribution of damage did not appear to be random, but instead showed a tendency for clustering amongst damaged cells, possibly indicating that cells damaged by the bystander signal may then release a further signal, leading to a sustained chain reaction.

One advantage of using a microbeam is that it is possible to selectively target either the cell nucleus or the cytoplasm. Shao and colleagues [14] have used the GCI particle microbeam to study the induction of micronuclei induced in a population of T98G glioma cells, after targeting the cytoplasm of one cell close to the centre of the population. They find that the overall yield of micronuclei increased from $13.5 \%$ in the non-irradiated control experiments, to $18.3 \%$ when the cytoplasm of one cell was irradiated with a single targeted ${ }^{3} \mathrm{He}^{2+}$ ion. Furthermore, no increase in the yield of micronuclei was seen when greater fractions of cells were targeted through their cytoplasm (either one cell, ten cells, or the whole population). In another experiment, AG01522 (AG0) primary human fibroblasts were co-cultured in alongside the T98G glioma cells in separate regions $5 \mathrm{~mm}$ apart. Targeting the cytoplasm of a single T98G cell with one ${ }^{3} \mathrm{He}^{2+}$ ion produced a $78 \%$ increase in the production of micronuclei within the non-irradiated AG0 population, demonstrating that bystander responses can be induced across genotypes.

There is now much interest in identifying the factors involved in mediating the bystander response. Shao et al. have used the GCI particle microbeam to 


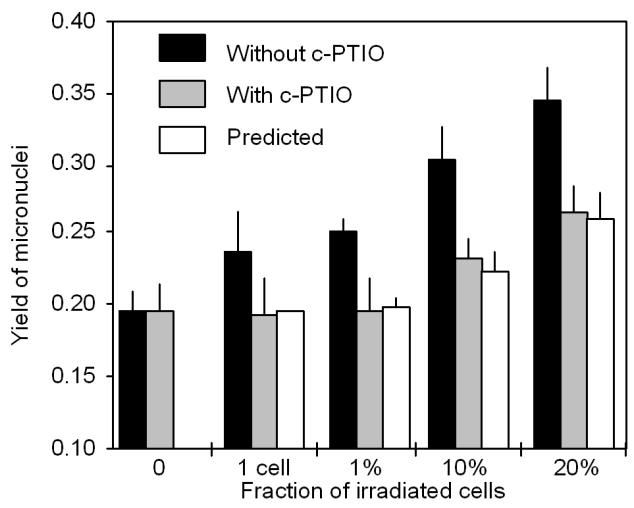

Fig. 3. Micronuclei yields where a fraction of the cells were irradiated with five ${ }^{3} \mathrm{He}^{2+}$ ions. With a nitric oxide specific scavenger present $(20 \mu \mathrm{m}$ c-PTIO), the yields of micronuclei are comparable to the predicted value, assuming no bystander effect. Data redrawn from [15].

investigate the role of nitric oxide-mediated signalling in the bystander response of individually targeted T98G glioma cells [15]. An exact fraction of the total number of cells (out of about 1200 cells) was irradiated with a single ${ }^{3} \mathrm{He}^{2+}$ ion and the production of micronuclei scored after one hour's incubation. The results show that the number of micronuclei induced rises sharply as the fraction of cells irradiated is increased from $0 \%$ to $20 \%$ of the total; about $28 \%$ of cells exhibit micronuclei when $20 \%$ of the cells are irradiated. However, if the experiment was repeated in the presence of c-PTIO (an NO-specific scavenger), it was found that the addition of the scavenger reduced the micronuclei yields to those expected if only direct effects were being produced (see Fig. 3), indicating that the bystander response had been inhibited when the NO signalling pathway was blocked.

\section{Conclusions}

The ability to micro-irradiate individual cells continues to be great use in understanding anomalous low-dose and non-targeted radiobiolical effects in cells. However, there is still a great deal that we do not know about the underlying mechanisms of these effects and further studies are required. It is clear however that our confidence in the linear no-threshold model of radiation risk is challenged by some of the findings arising from microbeam experiments. The key observations addressed here are; firstly, that a single helium ion through a single cell is sufficient to induce the full bystander response. Secondly, that the bystander response is a "binary" effect, such that a low dose will induce either no bystander effect, or the full effect. Thirdly, that the bystander response can be induced across genotypes. Fourthly, that targeting the cytoplasm can induce the bystander response, and finally, that NO is involved in the signalling pathway. 


\section{Acknowledgments}

The authors wish to acknowledge grants from the Cancer Research UK, the European Commission, and the U.S. Department of Energy.

\section{References}

[1] M. Folkard, B. Vojnovic, K.M. Prise, A.G. Bowey, R.J. Locke, G. Schettino, B.D. Michael, Int. J. Radiat. Biol. 72, 375 (1997).

[2] M. Folkard, G. Schettino, B. Vojnovic, S. Gilchrist, A.G. Michette, S.J. Pfauntsch, K.M. Prise, B.D. Michael, Radiat. Res. 156, 796 (2001).

[3] W.F. Morgan, Radiat. Res. 159, 567 (2003).

[4] K.M. Prise, M. Folkard, B.D. Michael, Radiat. Prot. Dosimetry 104, 347 (2003).

[5] H. Nagasawa, J.B. Little, Cancer Res. 52, 6394 (1992).

[6] W.F. Morgan, Radiat. Res. 159, 581 (2003).

[7] M. Folkard, B. Vojnovic, S. Gilchrist, K.M. Prise, B.D. Michael, Nucl. Instrum. Methods Phys. Res. B 210, 302 (2003).

[8] M. Sowa Resat, W.F. Morgan, Cancer Metastasis Rev. 23, 323 (2004).

[9] M. Folkard, B. Vojnovic, K.J. Hollis, A.G. Bowey, S.J. Watts, G. Schettino, K.M. Prise, B.D. Michael, Int. J. Radiat. Biol. 72, 387 (1997).

[10] S. Peng, M. Folkard, S. Gilchrist, R.J. Locke, Z. Yu, B.D. Michael, Nucl. Instrum. Methods Phys. Res. B 179, 145 (2001).

[11] K.M. Prise, O.V. Belyakov, M. Folkard, B.D. Michael, Int. J. Radiat. Biol. 74, 793 (1998).

[12] G. Schettino, M. Folkard, B.D. Michael, K.M. Prise, Radiat. Res. 163, 332 (2005).

[13] G. Schettino, M. Folkard, K.M. Prise, B. Vojnovic, K.D. Held, B.D. Michael, Radiat. Res. 505, 160 (2003).

[14] C. Shao, M. Folkard, B.D. Michael, K.M. Prise, Proc. Natl. Acad. Sci. 101, 13495 (2004).

[15] C. Shao, V. Stewart, M. Folkard, B.D. Michael, K.M. Prise, Cancer Res. 63, 8437 (2003). 